\title{
Design of Business Support Platform for Port Smart Power Utilization Facing to Alternative Maritime Power Supply Operation
}

\author{
Kun Huang ${ }^{1}$, Xiaoshu Huang ${ }^{1}$, Jian Huang ${ }^{1}$, Yingyuan Zhang ${ }^{1}$, Bin Yang ${ }^{2}$, \\ Wenjun Ruan ${ }^{2}$, Jingxia Cui ${ }^{2}$, Rutong Zhang ${ }^{1, a}$ \\ ${ }^{1}$ NARI Technology Co.,Ltd, Nanjing 211106,China, \\ ${ }^{2}$ State Grid Jiangsu Electric Power Company, Nanjing 211106,China, \\ a zhangrutong@sgepri.sgcc.com.cn
}

\begin{abstract}
Key words: alternative maritime Power (AMP); smart power utilization; business support platform Abstract: In this paper, the development status, application scenarios and development demands of alternative maritime power (AMP) in ports at home and abroad are studied and the outstanding significance of establishing the business support system for smart power utilization in port facing to AMP operation is analyzed. Aimed at the diversified needs of power utilization equipment and emerging demands of operational services with respect to the power grid in port, the scheme of the business support platform system facing to AMP and other smart power utilization equipment in port is prepared. The architecture of the platform system, the system's external interfaces, the extended functions in the future and other contents are mainly elaborated. On the basis of the development tendency of ports in China, the prospect concerning the application of the business support platform for smart power utilization in port is put forward.
\end{abstract}

\section{Introduction}

Port, the hub of land-and-water coordinated transport, is both an essential link in the transportation system of China and the essential economic resource of the state and local area. Modernized port in China will develop in the form of industrial parks with intelligent equipment in line with low carbon requirements, to respectively correspond to the changes and adjustment of the port's industrial development orientation, internal enterprise relationship and the relationship between the port and the nature. Following the globalization tendency of economy, the adjustment of global industrial structure and the extensive application of information technology, port gradually becomes intelligent, flexible, swift and refined, which drives the seamless connection and effective application of the port with each respective link. By the combination of port business, energy and information technology, the original operation management mode of the port will be changed, thus realizing intelligent production and management and refined energy application, providing differential green services, enhancing the role of the port in the supply chain and accomplishing the effective integration of port functions. With the increasingly emphasis of the world on energy conservation, emission reduction and environmental protection, the technology of serving anchored ships with the on-shore power supply system has become mature, which has been practically used by some ports abroad for the reduction of port environment pollution and is emphasized and promoted in domestic ports. Since 2000, America, Sweden, Netherlands Though the research on the AMP technology remains at the starting phase in China, since 2009, many domestic ports have established various AMP pilot engineering [1].

Current establishment of AMP facilities in port mainly focuses on the connection of power for ships berthing at port [2], but it is short of the support system for daily operation management of those AMP facilities and for the provision of operation business services. In addition, with the continuous expansion of the scale of port enterprise, the increasingly extensive application of the port power utilization system and the rising of the number thereof, many problems exist among 
various systems, making it hard to smoothly carry out the smart power utilization business in port. The business support system for smart power utilization in port is mainly to centrally manage and hierarchically control various port AMP equipment and auxiliary systems, thus supporting the operation business of port AMP under different application scenarios, and to satisfy other smart power utilization needs of the port, resolve the key problems of data integration, service sharing and process orchestration among systems, support the internal smart power utilization business of the port and provide value-added services for the society.

\section{Operation Demands of AMP}

Power utilization in port consists of production power, living power and auxiliary production power. Specifically, production power is mainly utilized for the operation of loading/unloading machinery, transportation machinery, warehousing equipment and the lighting of production sites, living power is supplied for office buildings, living facilities, etc., while auxiliary production power mainly includes maintenance power, public lighting power, auxiliary facility power, etc. With the low-carbon development in port, the demands of characteristic and diversified power become increasingly urgent. As an emerging business for port production, AMP has enriched the original forms of power utilization services with its extensive application and operation, but different types of ports will put forward different requirements on services. The operation of port AMP system can be divided into large coastal wharf, medium coastal and riverside wharfs and small inland and lake-side wharfs and based on the operation management ways of different wharfs, the operation service needs of AMP are verified.

1) The AMP system for large coastal wharfs and medium coastal and riverside wharfs is mainly to satisfy the power utilization of large ocean-going ships and large and medium transport ships for their loading and unloading when they are entering and berthing at the port. At the time, the smart power utilization platform supporting the operation of AMP has to accomplish many basic functions of power utilization information query and bill settlement between the shipping agency and the port; meanwhile, by means of information exchange with the port production scheduling system, it has to ensure process management, equipment monitoring, power utilization statistics, charge management, statistical analysis and other functions about AMP utilization of ships.

2) The AMP system for small inland and lake-side wharfs is mainly dedicated to the power utilization of medium and small inland ships for their loading and unloading during their berthing. Since such kinds of ships are generally operated by individuals, the business platform for smart power utilization supporting AMP operation needs to realize unmanned management of the entire business operation process; at the same time, business processing and settlement can be fulfilled by special means (such as swiping card, mobile phone APP and network).

3) The last category faces to the power utilization of emerging ports and can satisfy the operational service demands of different types of power utilization. Besides of the support to the operation of port AMP, the business support platform for smart power utilization also needs to take into full account of the operation management demands of distributive energy access, electric automobile, energy efficiency management equipment, power quality monitoring and other new types of power utilization services in port. In addition, with respect to the transformation of the port from a traditional energy consumer to an energy service provider, the business support platform has 
to possess the ability to explore new power utilization business and expand corresponding development space.

\section{Platform Design Scheme}

\section{System architecture}

The logical architecture of the support platform system for smart power utilization in port[3-4].
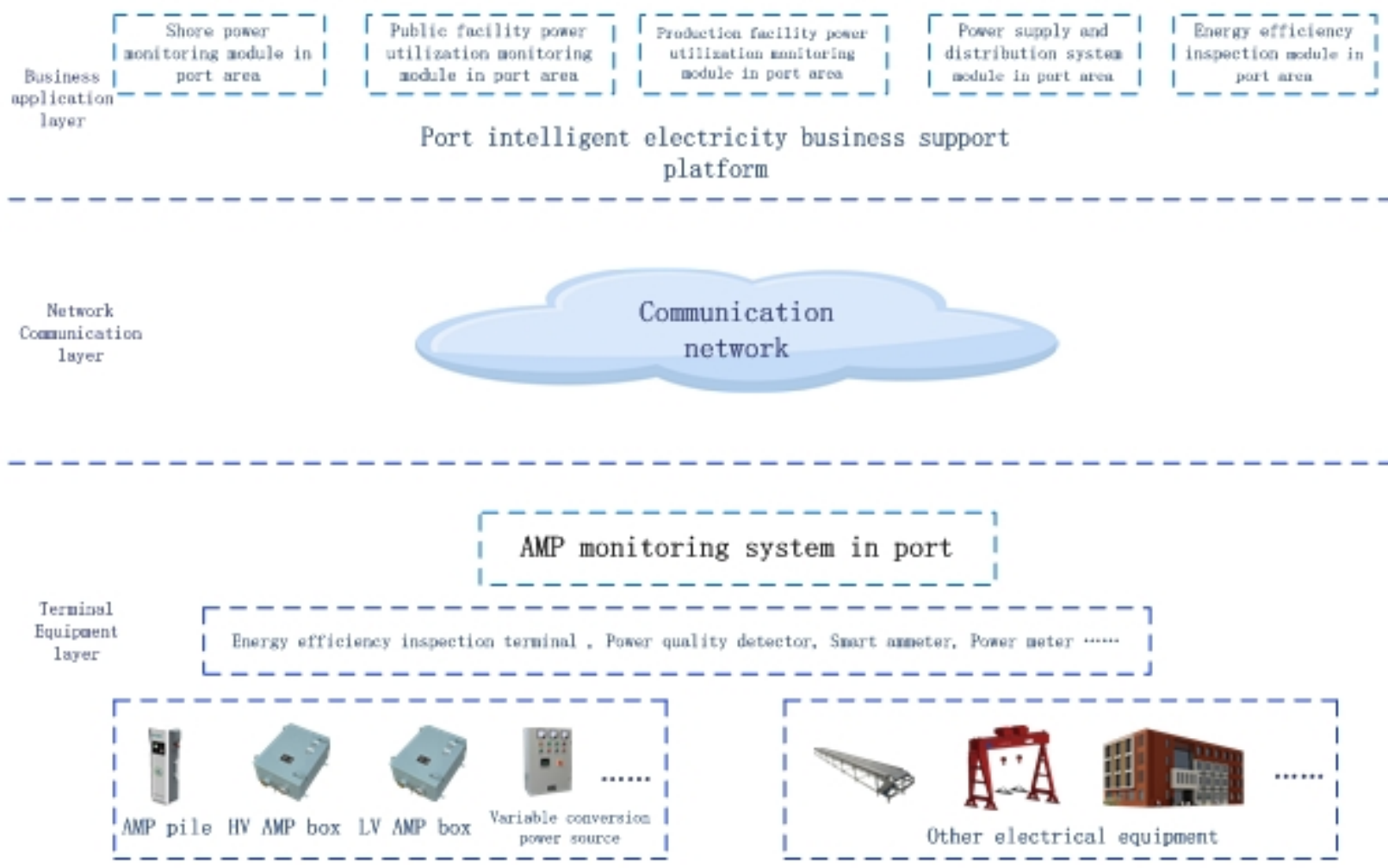

Fig. 1 System Logical Architecture of Business Support System for Smart Power Utilization in Port

1) The terminal equipment layer consists of the AMP connection equipment at the wharf apron, main wharf production power utilization equipment and relevant acquisition monitoring devices, principally aiming at providing AMP for ships in port, as well as the information acquisition, status monitoring and operation information summary of AMP equipment. Specifically, the AMP equipment includes AMP socket box, AMP pile, controller, acquisition equipment, metering device, network transmission device, AMP monitoring system in port, etc. Among them, the acquisition equipment is mainly used for data acquisition, monitoring and transmission of the AMP connection devices, such as the smart ammeter, energy efficiency monitoring terminal and energy quality inspection meter, while the AMP monitoring system in port is mainly to summarize, monitor and upload the operational information of various power utilization equipment. The main function of this layer is to transmit, monitor and upload information among various local devices and is applicable to optical fiber, CAN, power line carrier communication, RS-232/485 and many other local communication modes.

2) The network communication layer is the fundamental path connecting AMP and other power utilization equipment at the wharf apron and the business support platform for smart power 
utilization in port. Internet technology is most frequently used and based on the equipment application environment, the communication modes of Ethernet, wireless or optical fiber are allowed.

3) Based on the business support system for smart power utilization in port, the business application layer will incorporate with existing production scheduling system, power distribution automation system in port area, lighting control system in port area and other business functions in port, so as to carry out and manage the smart power utilization business in port of AMP, public facility power and production facility power.

\section{Mode of system deployment}

Based on the scale of port wharf and the application scenarios of the AMP system, the deployment of the business support platform system for smart power utilization in port can be divided into central deployment and distributive deployment[5].

Specifically, central deployment is mainly applicable to the AMP operation equipment required of unmanned operation in inland service areas, channelization sections of the channel, etc. The business support platform can be deployed in provincial or prefecture-level AMP operation companies. Individual consumers are able to access the platform through the client end system, use the AMP equipment and pay by prepaid cards or individual mobile terminals. This mode is convenient for the central management of systems and devices within some regions, facilitates consumers to take use of all AMP equipment within the region and can be easily maintained with a short construction period as a whole.

Distributive deployment is mainly to satisfy the hierarchic management demands of large port wharfs and respectively one business support platform system will be provided for the port group and each wharf company. Among them, the system of the group company will take charge of the summary management and statistical analysis of the AMP operation and other smart power utilization businesses of its various affiliated wharfs, while the system for each wharf company is responsible for managing internal AMP and other smart power utilization businesses.

It is beneficial to the refined management of each wharf by distributive deployment. Since the system for each wharf is relatively independent, system safety can be substantially enhanced. Even if a single sub-system suffers faults, it will not affect the AMP and smart power operation of the entire port group. However, as for large port groups, the investment at the earlier stage is relatively high, with rigorous requirement on system integration.

\section{Mode of system communication}

The communication between the business support platform for smart power utilization and its terminal AMP equipment is mainly realized by Ethernet access. By means of CAN/RS-485, optical fiber communication module and other modes, terminal AMP equipment are accessed to the central controller or the AMP monitoring system in port, for equipment management and monitoring, as well as the acquisition and communication of equipment information. Besides, terminal equipment can directly exchange data with the business support platform by wireless communication. The architecture of communication network of the business support platform for smart power utilization is as shown in Fig.2. 


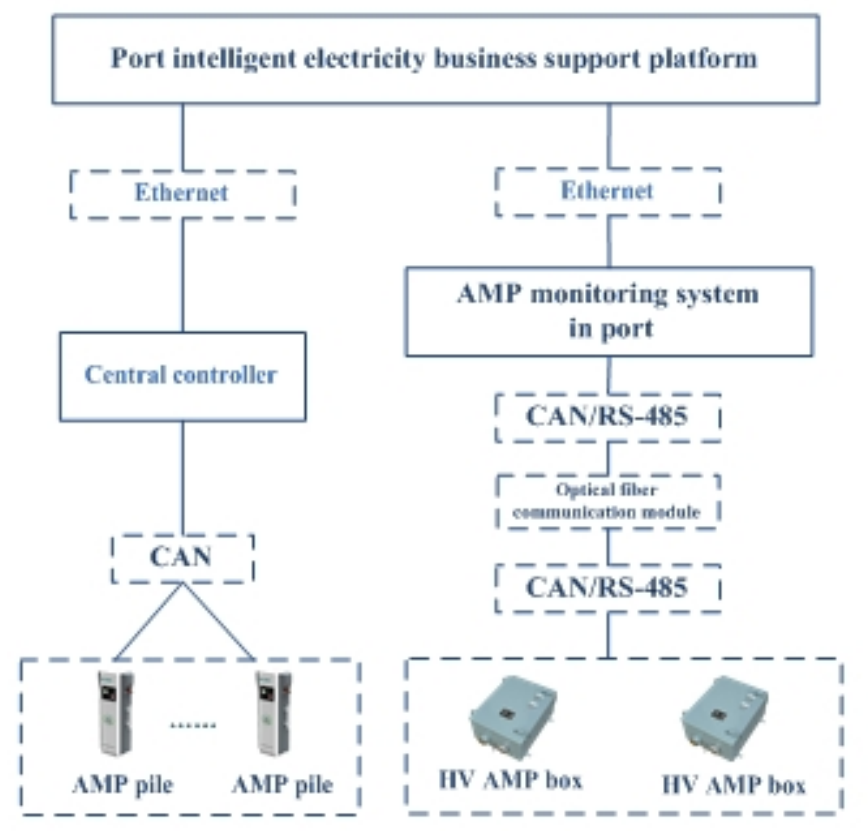

Fig. 2 Structure of Communication Network of Service Supporting Platform for Smart Power Consumption

\section{System interface}

Data exchange and mutual support are necessary for the effective operation of the business platform. Since interfaces between the platform and other business systems are needed for all data exchange and the realization of business application functions[6], the platform has to ascertain the data model, business process and interface models of interfaces with other system. Interfaces with several kinds of systems that are mainly associated with the business support platform for smart power utilization in port are introduced as follows:

1) Interface with the production scheduling system in port. The production scheduling system in port is mainly to provide the berthing information, ship parameters, the application of main production equipment (shore bridge, transtainer and conveyer belt) and other data about ships berthing at the port. The business support platform mainly takes use of these data for the operation, management and settlement of relevant services of AMP and ships berthing at the port.

2) Interface with the power grid automation system in port. The power grid automation system is mainly to provide main indicators, data and business information of the power grid in port, including the power supply system (clean energy power generation, substation, etc.), power distribution system (power distribution network in port area, distribution unit area) and power utilization system in port area. The business support platform mainly takes use of these data for the management and operation of AMP utilization, clean energy access and power utilization of main production equipment in the port area.

3) Interface with other non-production systems. In addition to the production system, there are many other systems for lighting management, office building management, electric shuttle bus management and many other kinds of non-production management. By virtue of the built-in external interfaces, these systems are able to provide data and information concerning power utilization services. The business support platform mainly takes use of these data for the 
management and operation of value-added services in port concerning smart power utilization.

\section{Extended Function of Platform Business}

The business support platform for smart power utilization in port facing to power utilization business and services in port, in addition to offering support to the AMP operation in port, can provide many value-added business functions such as public facility management, production facility management, power supply and distribution system management, energy efficiency monitoring and emission management of the port area. Based on the above functional system, the aid decision making and utilization optimization of power in the port area can be realized and the extended functions of the platform business are as shown in Fig.3.

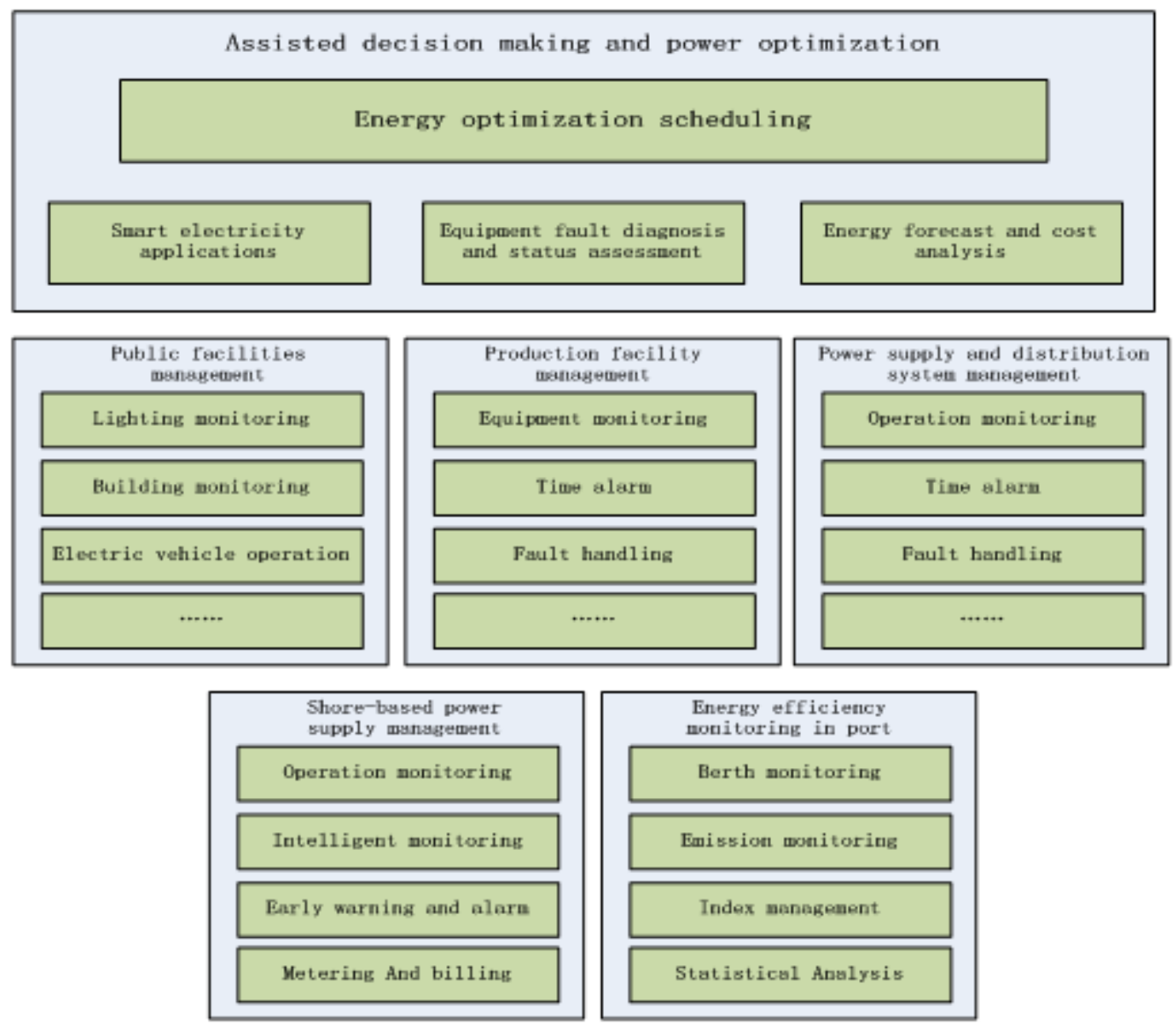

Port intelligent electricity business support platform

Fig. 3 Extended Function of Business Support Platform for Smart Power Utilization in Port

\section{Application Prospect of the Platform}

At present, the utilization business of AMP and other smart power in port is just at the starting phase, existing operational support systems in port mainly center around the application and monitoring of the production equipment and the port is lack of the support platform products facing to AMP operation and smart power utilization. Besides, with respect to different application 
scenarios of the AMP system, there is not an uniform business support platform for the operation to ensure the virtuous cycle and long-term development of AMP services in port. Considering the intelligent development tendency with low carbon in the form of industrial parks, the business support platform for smart power utilization in port is able to effectively bear the power utilization business needs during port development, expand power utilization services and explore the mode of value-added services. Now, the business support platform for smart power utilization in port facing to port AMP operation has been put into trial use and popularized in different port wharfs in Nanjing, Taizhou, Yancheng and Wuxi in Jiangsu.

\section{Conclusions}

The business support platform for smart power utilization in port is the informatization basis for realizing the operation, application and popularizing of AMP in port, and a key link in the construction of a smart port. By virtue of this platform, it is able to enhance and expand the existing power services in port, carry out new smart power services represented by AMP and effectively support a third party for the provision of value-added services of smart power utilization in port in later phase. It is beneficial for the smart integration of single systems in port, allows the access of terminal devices and operational systems, will promote information sharing and the application of the operation business within the port and among ports and boost the growth and large-scale application of the smart power utilization business in port.

\section{Acknowledgements}

This work was financially supported by the State Grid Jiangsu electric power company science and technology project (J2016007).

\section{References}

[1] TIAN Xin, YANG Liu, CAI Zhiyuan, et al. Summary of development of on-shore power supply for vessels technology in China and abroad [J]. Smart Grid, 2014, 2(11): 9-14 (in Chinese).

[2] CAO Shenghua, XU Dake, WANG Wenqiang, et al. Implementation of integrated solution of intelligent shore power supply system[J]. Huadian Technology, 2014, 2(11): 57-60 (in Chinese).

[3] HU Jiangyi, ZHU Enguo, DU Xingang, et al. Application status and development trend of power consumption information collection system $[\mathrm{J}]$. Automation of Electric Power Systems, 2014, 38(2): 131-135 (in Chinese).

[4] YAN Hongli, HE Jiasheng. Research and application of the electric vehicle charging facilities operation management system[J]. Electric Power IT, 2012, 10(9): 33-37(in Chinese).

[5] LIN Hongyu, ZHANG Jing, XU Kunpeng, et al. Design of interactive service platform for smart power consumption[J]. Power System Technology, 2011, 33(11): 255-259 (in Chinese).

[6] ZHANG Zhixiang. Design and Realization of Service Support System for Electric Vehicle Operation[D]. Beijing: Beijing University of Posts and Telecommunications, 2013 